\title{
BRIEF COMMUNICATION OPEN Metabolomic analysis of serum may refine 21-gene expression assay risk recurrence stratification
}

\author{
Amelia McCartney ${ }^{1}$, Alessia Vignoli $\mathbb{D}^{2,3}$, Leonardo Tenori $\mathbb{D i D}^{3,4}$, Monica Fornier ${ }^{5}$, Lorenzo Rossi $\mathbb{D D}^{1,6,7}$, Emanuela Risi ${ }^{1}$, \\ Claudio Luchinat ${ }^{2,3,8}$, Laura Biganzoli ${ }^{1}$ and Angelo Di Leo ${ }^{1}$
}

\begin{abstract}
Despite recent refinements to the 21-gene g score, allowing a better identification of patients who may derive no benefit from the addition of adjuvant chemotherapy to that of endocrine therapy, patients with early breast cancer still stand to be over-treated in the setting of clinical and/or genomic uncertainty or discordance. Here we describe and demonstrate a potential approach of further refining the OncotypeDX risk score by metabolomic analysis of serum. In a clinical dataset $(N=87)$, the risk of recurrence was further sub-stratified by metabolomic signature, with an effective splitting of each Oncotype risk classification. A total of seven recurrences were recorded, with metabolomic analysis accurately predicting six of these. Contrastingly, the genomic risk score of the seven recurrences ranged across all three Oncotype classifications (one recurrence occurred in the "low"-risk group, three in the "intermediate" group and three in the "high"-risk group).
\end{abstract}

npj Breast Cancer (2019)5:26; https://doi.org/10.1038/s41523-019-0123-9

\section{INTRODUCTION}

In meeting its primary endpoint of distant recurrent-free survival, the recently published TAILORx study demonstrated that adjuvant endocrine therapy was non-inferior to chemotherapy plus endocrine therapy in women with endocrine receptor-positive, HER2-negative early breast cancer (eBC) whose OncotypeDX 21gene expression assay risk recurrence scores (RS) was between 11 and $25 .^{1}$ Nevertheless, while many cases of eBC are cured by surgery \pm adjuvant endocrine therapy alone, a significant population are still over-treated due to the fear of recurrent disease established by clinicopathological and/or genomic risk factors. Genomic analysis of centrally derived tumour tissue assesses the potential, proportional benefit from adjuvant therapies, but surgery physically removes the factor (i.e. the primary tumour) upon which initial risk is estimated. Conversely, metabolomic analysis of serum detects the presence of residual micrometastatic disease, and is therefore a potential complementary tool with which to estimate residual risk of recurrence. ${ }^{2-7}$

Metabolomics is the -omic science that deals with the characterisation of the metabolome, in turn defined as the whole set of metabolites in a certain biological system such as a cell, a tissue, an organ or an entire organism. ${ }^{8}$ The two leading analytical techniques used to perform metabolomics are mass spectrometry (MS) and nuclear magnetic resonance (NMR) spectroscopy. Both techniques have their own strengths and limitations. MS overshadows NMR in terms of the number of compounds resolved, with a sensitivity in the range down to picomolar, requiring a very small volume of biospecimen. However, reproducibility remains its major limitation. ${ }^{9}$ Conversely, NMR analysis is high throughput and produces data that are highly reproducible and intrinsically quantitative, and thus more suitable for the fingerprinting analysis described here. ${ }^{8,10}$ Our group has already established a reproducible method of quantifying the individual metabolomic fingerprint, and its ability to accurately discriminate between advanced breast cancer and $\mathrm{eBC}^{2}$ Furthermore, we previously demonstrated that the metabolomic fingerprint can be used to predict the risk of disease recurrence in early disease, ${ }^{2-4}$ and that subsequent recurrence is characterised by higher (adjusted $P<0.05)$ serum levels of choline, phenylalanine, leucine, histidine, glutamate, glycine, tyrosine, valine, lactate and isoleucine. ${ }^{4}$

In this study, we retrospectively coupled NMR metabolomic predictions of recurrence with OncotypeDX RS in order to test the hypothesis that metabolomic prediction of risk recurrence could usefully split risk stratifications previously defined by OncotypeDX alone.

\section{RESULTS}

Metabolomic analysis

NMR spectra derived from the sera of 87 patients with $\mathrm{eBC}$ were compared with a matched population of 28 metastatic breast cancer ( $\mathrm{mBC}$ ) patients, previously analysed in a preceding study. ${ }^{3}$ In order to build a statistical model able to predict recurrence risk in eBC patients, 26 samples from patients with recurrence-free eBC and all $\mathrm{mBC}$ patients (training set) were compared using a Random Forest (RF) classifier. This model discriminated eBC with

\footnotetext{
1"Sandro Pitigliani” Department of Medical Oncology, Prato Hospital, Via Suor Niccolina 20, Prato, Italy; ${ }^{2}$ Consorzio Interuniversitario Risonanze Magnetiche di Metallo Proteine (C. I.R.M.M.P.), Via Sacconi 6, Sesto Fiorentino 50019, Italy; ${ }^{3}$ Centre for Magnetic Resonance (CERM), University of Florence, Via Sacconi 6, Sesto Fiorentino 50019, Italy; ${ }^{4}$ Department of Experimental and Clinical Medicine, University of Florence, Largo Brambilla 3, Florence 50100, Italy; ${ }^{5}$ Breast Medicine Service, Memorial Sloan-Kettering Cancer Center, Weill Cornell Medical College, New York, NY, USA; ${ }^{6}$ Institute of Oncology of Southern Switzerland (IOSI), Bellinzona, Switzerland; ${ }^{7}$ Breast Unit of Southern Switzerland (CSSI), Lugano, Switzerland and ${ }^{8}$ Department of Chemistry, University of Florence, Via della Lastruccia 3, Sesto Fiorentino 50019, Italy Correspondence: Angelo Di Leo (angelo.dileo@uslcentro.toscana.it)

These authors contributed equally: Amelia McCartney, Alessia Vignoli

These authors jointly supervised this work: Laura Biganzoli, Angelo Di Leo
}

Received: 12 February 2019 Accepted: 6 August 2019

Published online: 29 August 2019 


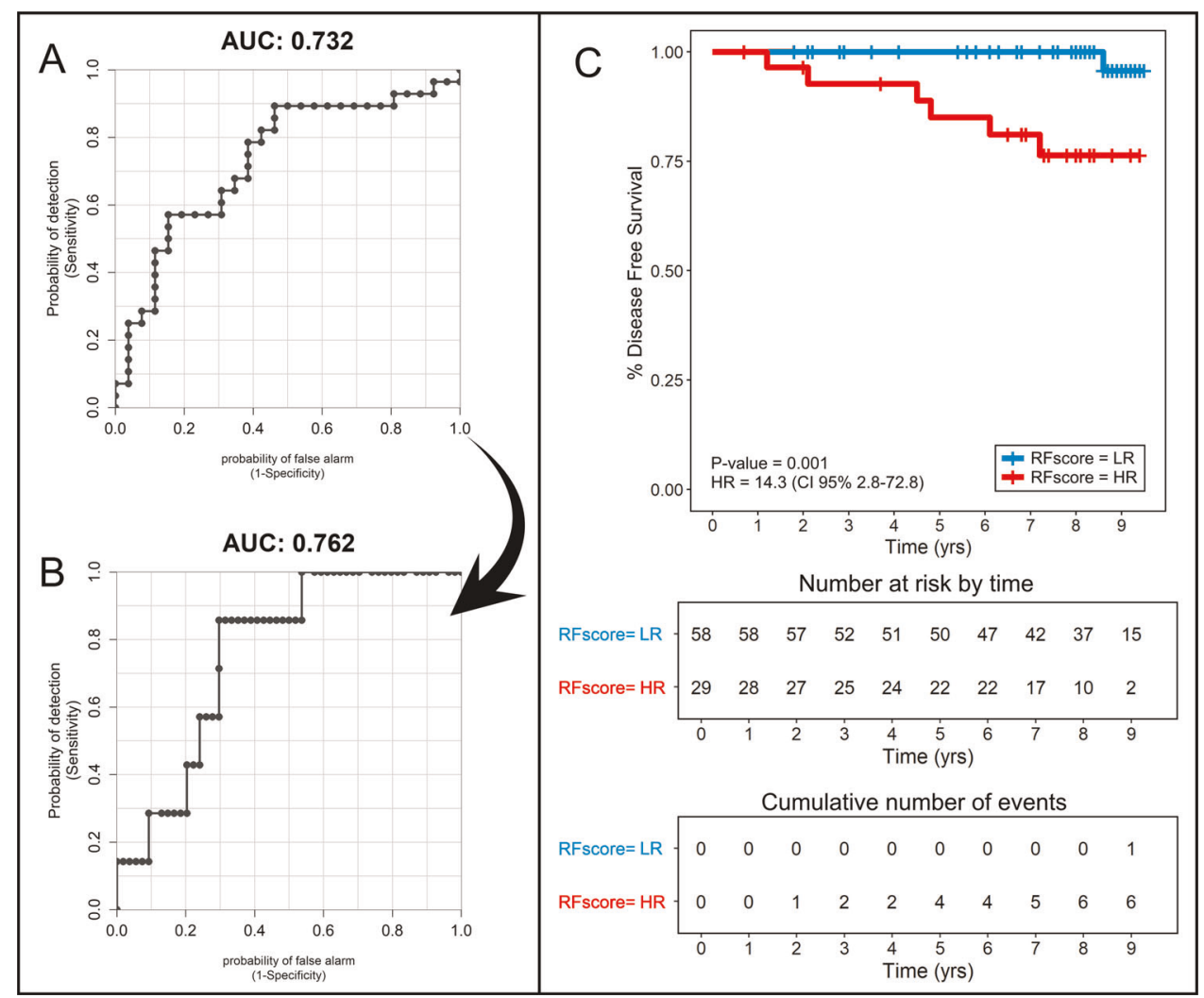

Fig. 1 Summarised results obtained by nuclear magnetic resonance (NMR) metabolomics: a area under the receiver operating characteristics curve (AUC) for the Random Forest (RF) model discriminating 26 early breast cancer (eBC) patients free from cancer recurrence at follow-up and 28 metastatic breast cancer ( $\mathrm{mBC}$ )-matched patients (training set). The score plotted is the RF risk score that expresses the probability that each sample included in the model has been classified correctly as eBC, or misclassified as mBC. $\mathbf{b}$ AUC for RF risk score of the validation set constituted by $7 \mathrm{eBC}$ patients who developed recurrent disease and $54 \mathrm{eBC}$ patients without recurrence. High RF risk score is deemed to represent a high risk of recurrence, as it means that the metabolomic fingerprint of an $\mathrm{eBC}$ patient is more closely resembles that of mBC. $\mathbf{c}$ Overall eBC patients, plotting actual disease-free survival over time (measured in years) according to estimated metabolomic risk score (Kaplan-Meier curves). "Low" (LR) and "High" (HR) RF risk patients are significantly clustered with a $P$ value of 0.001 (calculated with log-rank test) and a hazard ratio of 14.3. Censored events represent either the time of last recorded clinical follow-up or time of disease recurrence. Timing of recurrent disease events is separately presented in the lower-most risk table

respect to $\mathrm{mBC}$ patients with an area under the receiver-operating characteristics (ROC) curve (AUC) of 0.732 (Fig. 1a), allowing us to obtain an RF risk score for each eBC patient. This model was tested by analysing all remaining $\mathrm{eBC}$ patients (validation set: 54 relapsefree patients and 7 with relapse), hypothesising that a metabolomic signature similar to that of $\mathrm{mBC}$ patients would be predictive of cancer recurrence. In the validation set, eBC patients without relapse were clustered with respect to relapsed patients with an AUC of 0.762 (Fig. 1b), demonstrating the predictive strength of our statistical model. Furthermore, analysing the RF risk score of all $\mathrm{eBC}$ (both training and validation) with Kaplan-Meier curves (Fig. 1c), a clear discrimination is evident between patients without disease recurrence and those with relapse, demonstrated by a $P$ value of 0.001 and a hazard ratio (HR) of 14.3.

The metabolomic RF risk score was then combined with the predictive strength of the OncotypeDX assay, with the hypothesis that the metabolomic score could sub-classify each Oncotypedefined risk class into two subgroups: low and high risk, according to the threshold $(\mathrm{RF} \geq 53)$ determined in our previous study. ${ }^{3}$ Furthermore, by utilising RF $>69$, an optimised threshold for this new dataset, improved results were obtained in the low and intermediate Oncotype risk classes (Fig. 2). In line with the landmark TAILORx study, ${ }^{1}$ the classification ranges of RS defined by that study are reported here.

The metabolomic RF risk score successfully split each Oncotype risk level, consequently refining genomic risk prediction. In the genomic "low-risk" group (defined by a RS $\leq 10$ ), all patients with a corresponding low RF risk score were disease free at follow-up. Among the seven patients classified as high risk by RF, one relapse occurred. Within the Oncotype "intermediate-risk" class (RS 11-25), two relapses occurred in the "high-risk" RF subgroup and one in the "low-risk" subgroup. Notably, metabolic RF scores within the Oncotype "intermediate" group were heterogeneously distributed, suggesting variability in risk existing within that otherwise uniform stratification. In the "high-risk" Oncotype group $(\mathrm{RS} \geq 26)$, all relapsed patients were correctly classified at "high risk" by metabolomic RF score.

\section{DISCUSSION}

The TAILORx trial protocol conservatively enriched one-third of its "intermediate" RS group (deemed by investigators as a RS between 11 and 25) by including 2373 patients defined as being at low risk of recurrence by classical OncotypeDX parameters ("low" risk is expressed by OncotypeDX as a RS <18). Furthermore, patients who fell into the top end of OncotypeDX-defined "intermediate-risk" group (RS 26-30) were allocated to the TAILORx-redefined "high-risk" group. It is perhaps therefore unsurprising that TAILORx found no additional benefit for chemoendocrine therapy in the revised "intermediate/mid-range" group, as a significant proportion of those assigned likely never stood to benefit from chemotherapy. An additional consequence of shifting patients with a RS between 26 and 30 to a "high-risk" 


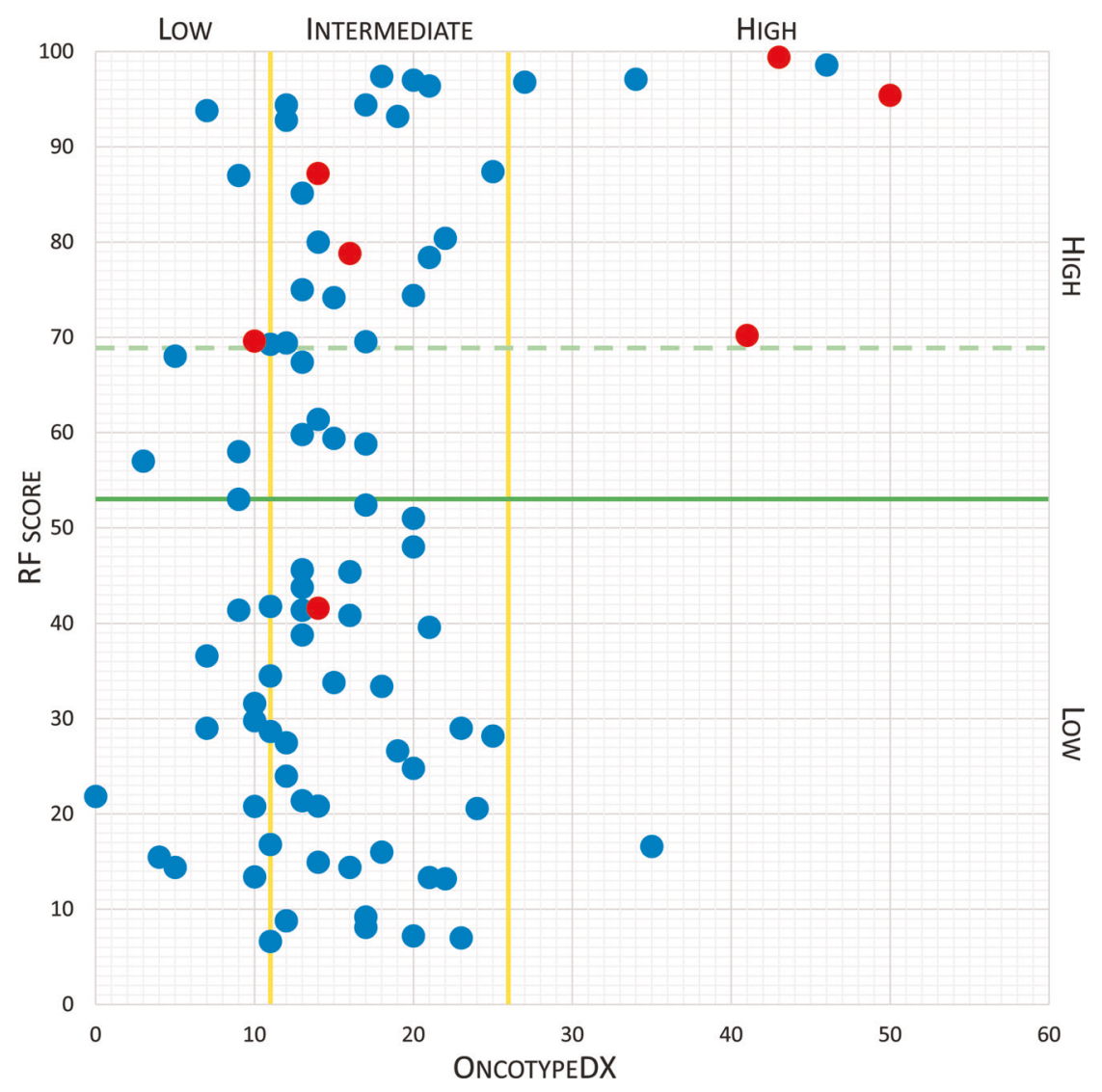

Fig. 2 OncotypeDX score plotted against metabolomic Random Forest (RF) score. The predicted outcome based on the TAILORx-defined recurrence score classification (low/intermediate/high), sub-stratified by nuclear magnetic resonance (NMR) metabolomic RF risk score (low/ high), compared to actual patient outcomes (recurrences denoted in red). The dashed line represents the cut-off for the metabolomic RF score optimised in this dataset

group (all of whom received protocol-mandated chemoendocrine therapy) was the loss of any chance of detecting a subgroup within that classification who might safely avoid chemotherapy. Exploratory analyses of patients aged $\leq 50$ suggested there may be benefit from chemotherapy if the RS was at the upper end of the TAILORx "mid-range" (RS 16-25), although this may be possibly attributed to the secondary ovarian suppression effected by chemotherapy in a largely pre-menopausal cohort, rather than a direct benefit of cytotoxic therapy itself. TAILORx did not identify any other factors that stratified risk of recurrence within the Oncotype-defined "intermediate-risk" group. Harnessing metabolomic analysis may potentially further this endeavour.

While exploratory in nature, this analysis represents a new integration of prognostic information derived centrally from the primary tumour, with aberrant metabolomic signalling from the periphery, which persists in the presence of micrometastatic disease. OncotypeDX RS stratifications were split further by metabolomic analysis, with all-but-one recurrences falling in "high-risk" metabolomic sets. The only patient with subsequent recurrence who was incorrectly classified by metabolomic analysis as having a "low risk" of recurrence developed cutaneous metastases $\sim 8.5$ years after initial diagnosis, on a background of having completed adjuvant anthracycline and taxane-containing chemotherapy and 5 years of endocrine therapy. This raises the question as to whether metabolomic analysis of specimens collected at the time of initial diagnosis may be limited in detecting signals of micrometastatic disease with a long lead time to clinical manifestation, or if more indolent subtypes of metastatic disease may evade metabolomic detection. The strength of this approach may be underestimated by this study, given the small number of disease recurrence events observed in the studied cohort, which was largely comprised of patients with luminal A-like disease, which often relapses beyond 5 years of completing adjuvant therapy. ${ }^{11}$ More relapses may yet occur in time, particularly in those patients whose metabolomic score placed them at higher risk of doing so. The results of this study require validation in a larger patient cohort.

\section{METHODS}

Patient data and sample collection

Serum samples were selected from a breast cancer biobank belonging to Memorial Sloan Kettering Cancer Center (MSKCC), derived from patients with $\mathrm{eBC}$ who provided prospective written informed consent for the collection of serum and clinical information for future research purposes. Approval was obtained according to a protocol ratified by the ethics committee of MSKCC. Samples were collected post-operatively, between June 2007 and December 2009, with a mean follow-up from diagnosis of 7 years (range, 1-9 years). MSKCC maintained a database of all clinicopathological data and clinical outcomes, which was provided in deidentified form to the collaborating group in Italy. Study serum samples $(500 \mu \mathrm{l})$ were maintained at $-80^{\circ} \mathrm{C}$ from collection until transfer over dry ice from MSKCC to Italy, where they were again stored at $-80^{\circ} \mathrm{C}$ until analysis. Serum samples were anonymised prior to transfer.

Of the entire MSKCC dataset $(N=139)$, all available samples from patients with early oestrogen receptor-positive/progesterone receptorpositive $(\mathrm{PR}+) / \mathrm{HER} 2$-negative disease were selected for analysis. In the interests of examining as homogeneous a population as possible, samples from patients with PR-negative disease were excluded. Similarly, as OncotypeDX is not validated in patients with HER2-positive disease, HER2-positive samples were not included in this analysis. To reduce the risk of detecting a metabolomic "false-positive" signal, samples from patients 
who developed subsequent second primary malignancies, or a second primary breast cancer, were also excluded. The baseline characteristics of the patients $(N=87)$ included in this analysis are presented in Supplementary Table $1 .{ }^{12}$ Just under one-half $(48 \%)$ of subjects were aged 50 years or less. Primary tumours measuring between 1.1 and $2 \mathrm{~cm}$ (T1c) represented $48 \%$ of the studied cohort. Although $87 \%$ of samples were from patients with node-negative disease, $80 \%$ had primary disease pathologically classed as either grade 2 or 3 .

\section{${ }^{1} \mathrm{H}$-NMR sample analysis}

Samples were prepared following the standard protocols detailed by Bernini et al. ${ }^{13}$ Frozen serum samples were thawed at room temperature and shaken before use, then a total of $350 \mu \mathrm{L}$ of a sodium phosphate buffer $\left(10.05 \mathrm{~g} \mathrm{Na}_{2} \mathrm{HPO}_{4} \cdot 7 \mathrm{H}_{2} \mathrm{O} ; 0.2 \mathrm{~g} \mathrm{NaN} ; 0.4 \mathrm{~g}\right.$ sodium trimethylsilyl $\left[2,2,3,3-{ }^{2} \mathrm{H}_{4}\right]$ propionate in $500 \mathrm{~mL}$ of $\mathrm{H}_{2} \mathrm{O}$ with $\left.20 \%(\mathrm{v} / \mathrm{v}){ }^{2} \mathrm{H}_{2} \mathrm{O} ; \mathrm{pH} 7.4\right)$ was added to $350 \mu \mathrm{L}$ of each serum sample, and the mixture was homogenised by vortexing for $30 \mathrm{~s}$. A total of $600 \mu \mathrm{L}$ of this mixture was transferred into a $5.0 \mathrm{~mm}$ NMR tube for the analysis.

One-dimensional ${ }^{1} \mathrm{H}$-NMR spectrum for all samples of eBC patients were acquired using a Bruker $600 \mathrm{MHz}$ spectrometer (Bruker BioSpin) operating at $600.13 \mathrm{MHz}$ proton Larmor frequency and equipped with a $5 \mathrm{~mm}$ PATXI ${ }^{1} \mathrm{H}-,{ }^{13} \mathrm{C}-{ }^{15} \mathrm{~N}$ - and ${ }^{2} \mathrm{H}$-decoupling probe, including a $z$-axis gradient coil, an automatic tuning matching and an automatic and refrigerated $\left(6^{\circ} \mathrm{C}\right)$ sample changer (SampleJet). A BTO 2000 thermocouple served for temperature stabilisation at the level of $\sim 0.1 \mathrm{~K}$ at the sample. Before measurement, samples were kept for at least $5 \mathrm{~min}$ inside the NMR probehead, for temperature equilibration at $310 \mathrm{~K}$. Water-suppressed Carr-Purcell-Meiboom-Gill (CPMG) ${ }^{14}$ spin echo pulse sequence (RD- $90^{\circ}$ $\left(\tau-180^{\circ}-\tau\right)_{n}$-acq) with a total spin echo $(2 n \tau)$ of $80 \mathrm{~ms}$ was used in order to obtain one-dimensional ${ }^{1} \mathrm{H}$-NMR spectra in which broad signals from highmolecular-weight metabolites are attenuated. Eighty FIDs were collected into 73,728 data points over a spectral width of $12,019 \mathrm{~Hz}$, with a relaxation delay of $4 \mathrm{~s}$ and acquisition time (acq) of $3.1 \mathrm{~s}$. One-dimensional ${ }^{1} \mathrm{H}-\mathrm{NMR}$ CPMG spectra of serum samples from patients with metastatic hormone receptor-positive, HER2-negative breast cancer were retrieved from a preexisting MSKCC cohort analysed in a previous study already published by our group. ${ }^{3}$

Free induction decays were multiplied by an exponential function equivalent to a $1.0 \mathrm{~Hz}$ line-broadening factor before applying Fourier transformation. Transformed spectra were automatically corrected for phase and baseline distortions and calibrated (anomeric glucose doublet at $5.24 \mathrm{ppm}$ ) using TopSpin 3.2 (Bruker Biospin srl).

Each 1D spectrum in the range $0.2-10.00 \mathrm{ppm}$ was segmented into $0.02 \mathrm{ppm}$ chemical shift bins and the corresponding spectral areas were integrated using the AMIX software (version 3.8.4, Bruker BioSpin). Binning is a means to reduce the number of total variables, to compensate for subtle signal shifts and to filter noise in the spectra, making the analysis more robust and reproducible. ${ }^{15,16}$ The region between 4.0 and $6.0 \mathrm{ppm}$ containing the residual water signal was removed and the dimension of the system was reduced to 356 bins. Total area was used as normalisation method on the data prior to pattern recognition.

\section{Statistical analysis}

Data analyses were performed using the open source software $R$. The statistical approach successfully utilised in our previous papers ${ }^{3,4}$ to predict the risk of disease recurrence was again applied in this study. The NMR data of the groups of $87 \mathrm{eBC}$ and $28 \mathrm{mBC}$ patients was randomly split into two independent cohorts: a training set consisting of $26 \mathrm{eBC}$ patients recurrence free after a mean of 7 years of follow-up, and all $\mathrm{mBC}$ patients, plus a validation set consisting of all remaining $\mathrm{eBC}$ patients ( 54 free of recurrence and 7 with recurrent disease).

The initial analysis was restricted to the training set, with the first step to confirm that metabolomic fingerprints could distinguish between eBC patients without recurrence and $\mathrm{mBC}$ patients. For this purpose, a RF classifier ${ }^{17}$ was built. RF is a classification algorithm that uses an ensemble of unpruned decision trees (forest), each of which is built on a bootstrap sample of the training data using a randomly selected subset of variables (bins). ${ }^{18,19}$ The percentage of trees in the forest that assign one sample to a specific class can be inferred as a probability of belonging to a given class. ${ }^{3}$ In our case, each tree was used to predict whether a sample represents early or metastatic disease. For each eBC patient, a score was created that expresses the extent to which the serum metabolomic profile appeared to be similar to the profile of a confirmed metastatic sample, designated as the "RF risk score." This score is based on the percentage of trees in the ensemble that misclassify a sample from a patient with $\mathrm{eBC}$ as belonging to the cohort of $\mathrm{mBC}$ patients. For all calculations, the R package " $\mathrm{RF}^{\text {"17 }}$ was used to grow a forest of 500 trees, using the default settings, and ROC analysis was used to evaluate the performance of the model.

The final step was to test the hypothesis that a metabolomic signature similar to that of $\mathrm{mBC}$ patients would prove truly predictive of cancer recurrence. Using ROC analysis, the performance of the RF risk scores was compared with actual clinical outcome. ${ }^{12}$ To delineate metabolomic high risk of relapse, the cut-off for the RF score optimized in our previous study $^{3}$ ( $R F \geq 53$ ) was adopted. It is worth of noting that using RF $>69$ improved results can be obtained in the "low" and "intermediate" Oncotype risk classes, this optimised threshold for this new dataset was determined using the function "coords" of the R package "pROC" that maximised the Youden's $J$ statistic. ${ }^{20}$ The ability of the RF risk score to predict breast cancer recurrences was also assessed using Kaplan-Meier curves, with additional calculation of the HR and $P$ value assessed by logrank test.

\section{Reporting summary}

Further information on research design is available in the Nature Research Reporting Summary linked to this article.

\section{DATA AVAILABILITY}

Analysed metabolomics datasets used to derive Figs 1 and 2 of the published article are publicly available in the figshare repository https://doi.org/10.6084/m9. figshare.8982221. ${ }^{12}$ Raw NMR spectra data (raw metabolomics data) supporting Figs 1 and 2 of the published article can be accessed from the corresponding author on reasonable request. Clinical (patient) data (supporting Supplementary Table 1 of the published article) are not publicly available, but can be accessed from the corresponding author on reasonable request as described at https://doi.org/ $10.6084 / \mathrm{m}$.figshare.8982221. ${ }^{12}$ The data generated and analysed during this study are described in the following data record: https://doi.org/10.6084/m9. figshare.8982221. ${ }^{12}$

\section{ACKNOWLEDGEMENTS}

This work was supported by a grant from the Breast Cancer Research Foundation, New York USA (grant number BCRF 18-054, jointly to L.B. and M.F.). A.V. is supported by an AIRC fellowship for Italy. We acknowledge the support and the use of resources of Instruct-ERIC, a landmark ESFRI project and specifically the CERM/CIRMMP Italy Centre.

\section{AUTHOR CONTRIBUTIONS}

A.M.C., A.V., L.T., M.F., L.R., E.R., C.L., L.B. and A.D.L. conceived this study; M.F. provided biobank samples and anonymised patient data; A.V., L.T. and C.L. performed metabolomic analyses, and all authors interpreted the subsequent results. A.M.C. and A.V. drafted the initial manuscript, which all authors subsequently revised. All authors approved the final version, and all have agreed to be accountable for all aspects of the work.

\section{ADDITIONAL INFORMATION}

Supplementary information accompanies the paper on the npj Breast Cancer website (https://doi.org/10.1038/s41523-019-0123-9).

Competing interests: A.D.L. has received honoraria for attending advisory boards and as a speaker at sponsored symposia by Genomic Health. The other authors declare no competing interests.

Publisher's note: Springer Nature remains neutral with regard to jurisdictional claims in published maps and institutional affiliations.

\section{REFERENCES}

1. Sparano, J. A. et al. Adjuvant chemotherapy guided by a 21-gene expression assay in breast cancer. N. Engl. J. Med. 379, 111-121 (2018).

2. Oakman, C. et al. Identification of a serum-detectable metabolomic fingerprint potentially correlated with the presence of micrometastatic disease in early breast cancer patients at varying risks of disease relapse by traditional prognostic methods. Ann. Oncol. 22, 1295-1301 (2011). 
3. Tenori, L. et al. Serum metabolomic profiles evaluated after surgery may identify patients with oestrogen receptor negative early breast cancer at increased risk of disease recurrence. Results from a retrospective study. Mol. Oncol. 9, 128-139 (2015).

4. Hart, C. D. et al. Serum metabolomic profiles identify ER-positive early breast cancer patients at increased risk of disease recurrence in a multi-center population. Clin. Cancer Res. 23, 1422-1431 (2017).

5. Claudino, W. M. et al. Metabolomics: available results, current research projects in breast cancer, and future applications. J. Clin. Oncol. 25, 2840-2846 (2007).

6. Hart, C. D., Tenori, L., Luchinat, C. \& Di Leo, A. Metabolomics in breast cancer: current status and perspectives. Adv. Exp. Med. Biol. 882, 217-234 (2016).

7. McCartney, A. et al. Metabolomics in breast cancer: a decade in review. Cancer Treat. Rev. 67, 88-96 (2018)

8. Vignoli, A. et al. High-throughput metabolomics by 1D NMR. Angew. Chem. Int. Ed. Engl. 58, 968-994 (2019).

9. Vignoli, A. et al. NMR-based metabolomics identifies patients at high risk of death within two years after acute myocardial infarction in the AMI-Florence II cohort. BMC Med. 17, 3 (2019).

10. Savorani, F., Rasmussen, M. A., Mikkelsen, M. S. \& Engelsen, S. B. A primer to nutritional metabolomics by NMR spectroscopy and chemometrics. Food Res. Int 54, 1131-1145 (2013).

11. Pan, $H$. et al. 20-Year risks of breast-cancer recurrence after stopping endocrine therapy at 5 years. N. Engl. J. Med. 377, 1836-1846 (2017).

12. McCartney, A. et al. Metabolomic analysis of serum may refine 21-gene expression assay risk recurrence stratification. Figshare Dataset https://doi.org/10.6084/ m9.figshare.8982221 (2019).

13. Bernini, P. et al. Standard operating procedures for pre-analytic handling of blood and urine for metabolomic studies and biobanks. J. Biomol. NMR 49, 231-243 (2011).
14. Meiboom, S. \& Gill, D. Modified spin-echo method for measuring nuclear relaxation times. Rev. Sci. Instrum. 29, 688-691 (1958).

15. Spraul, M. et al. Automatic reduction of NMR spectroscopic data for statistical and pattern recognition classification of samples. J. Pharm. Biomed. Anal. 12, 1215-1225 (1994).

16. Holmes, E. et al. Automatic data reduction and pattern recognition methods for analysis of ${ }^{1} \mathrm{H}$ nuclear magnetic resonance spectra of human urine from normal and pathological states. Anal. Biochem. 220, 284-296 (1994).

17. Breiman, L. Random forests. Mach. Learn. 45, 5-32 (2001).

18. Touw, W. G. et al. Data mining in the life sciences with random forest: a walk in the part or lost in the jungle? Brief. Bioinform. 14, 315-326 (2013).

19. Verikas, A., Gelzinis, A. \& Bacausekiene, M. Mining data with random forests: a survey and results of new tests. Pattern Recognit. 44, 330-349 (2011).

20. Youden, W. J. Index for rating diagnostic tests. Cancer 3, 32-35 (1950).

(i) Open Access This article is licensed under a Creative Commons Attribution 4.0 International License, which permits use, sharing, adaptation, distribution and reproduction in any medium or format, as long as you give appropriate credit to the original author(s) and the source, provide a link to the Creative Commons license, and indicate if changes were made. The images or other third party material in this article are included in the article's Creative Commons license, unless indicated otherwise in a credit line to the material. If material is not included in the article's Creative Commons license and your intended use is not permitted by statutory regulation or exceeds the permitted use, you will need to obtain permission directly from the copyright holder. To view a copy of this license, visit http://creativecommons. org/licenses/by/4.0/.

(c) The Author(s) 2019 